\title{
A Survey of Recent Developments in Cryptographic Algorithms for Smart Cards
}

\author{
Bart Preneel ${ }^{1}$ \\ Dept. Electrical Engineering-ESAT/COSIC, K.U.Leuven \\ Kasteelpark Arenberg 10, B-3001 Leuven, Belgium
}

\begin{abstract}
This article presents an update on recent developments in the area of cryptographic algorithms that are relevant for smart cards. It includes a review of the status of hash functions, block ciphers and stream ciphers and presents update on authenticated or unforgeable encryption. Finally the issue of secure padding for the RSA algorithm is discussed and the status of Elliptic Curve Cryptography is briefly reviewed.
\end{abstract}

Key words: cryptography, smart cards PACS: 89.20.Ff

\section{Introduction}

During the last decade, the computational power available on smart cards has increased significantly. Public-key smart cards are now widely deployed for payment applications (e.g., EMV). GSM operators have rolled out worldwide more than 1 billion SIM (Subscribe Identity Module) cards, and similar cards are being deployed in 3GSM (under the name USIM cards); both of these are restricted to symmetric cryptography. A third major application of smart card is pay television. On the other hand, smart cards for electronic signatures have not become popular with the exception of the electronic identity cards that are being rolled out by governments.

1 This author's work was supported in part by the Concerted Research Action (GOA) Ambiorics 2005/11 of the Flemish Government and in part by the European Commission through the IST Programme under Contract IST-2002-507932 ECRYPT. 
In 2001, Borst et al. have provided an overview of cryptographic algorithms used in smart cards [19] and of attacks on these algorithms. Since then, our understanding of cryptographic algorithms has improved significantly. The most remarkable developments are the attacks on the hash functions MD5 and SHA-1 and the new attacks on generic hash function constructions. Less visible but equally important has been the progress in the cryptanalysis of stream ciphers such as A5/1 (GSM) and E0 (Bluetooth). In the area of block ciphers, the adoption of the AES has shifted the attention from block ciphers towards their modes of use, and in particular modes for authenticated or unforgeable encryption. In the area of public key cryptology fewer developments can be reported, but the security of padding modes for RSA remains an important issue.

The article is organised as follows. In Section 2 we present an update on hash functions, stream ciphers, block ciphers and their modes. Section 3 focuses on public key algorithms and Section 4 presents the conclusions.

\section{Symmetric Primitives}

In this section, we discuss the following symmetric primitives: hash functions, MAC algorithms, block ciphers, stream ciphers, and modes for authenticated (or unforgeable) encryption.

\subsection{Hash Functions}

Cryptographic hash functions are a widely deployed primitive for message authentication. They compress strings of arbitrary lengths to strings of fixed lengths (typically between 128 and 256 bits). Cryptographic hash functions need to satisfy the following security properties [63,72]:

- preimage resistance: it should be hard to find a preimage for a given hash result;

- 2nd preimage resistance: it should be hard to find a 2nd preimage for a given input;

- collision resistance: it should be hard to find two different inputs with the same hash result.

For an ideal hash function with an $n$-bit result, finding a (2nd) preimage requires approximately $2^{n}$ hash function evaluations (for this section one hash function evaluation will be the time unit). On the other hand, finding a collision requires time only $2^{n / 2}$; this follows from the birthday paradox, which 
is based on the fact that a set of $r$ elements contains $r(r-1) / 2$ pairs of elements (and hence if there are 23 people in a room, the probability that at least two of them have the same birthday is larger than 50\%). Collision resistance implies 2 nd preimage resistance, but the formal relation between these definitions is more complex and subtle than one would expect (see Rogaway and Shrimpton [78]).

The best known application of hash functions is digital signature schemes, in which one signs the short hash value of a message rather than the message itself. This results in shorter signatures and more efficient signature schemes; in addition, the hash function is required to behave as a random mapping and in this way destroy the algebraic structure that may be present in the signature scheme (see also Sect. 3). For digital signature schemes, collision resistance is important: if the signer or the verifier can find collisions, they have obtained digital signatures on different messages, which clearly can create problems in the case of a dispute.

Other applications of hash functions include hashing of passphrases (this requires preimage resistance) and commitment to a string (this requires collision resistance). The derivation of keys and the HMAC construction (cf. Section 2.2) require that the hash function is pseudo-random if part of its input is keyed.

Most practical hash functions are iterated hash functions: they are based on a compression function $f$ that hashes fixed size inputs. The input string $X$ is first padded to a string which has a length that is a multiple of the block length; for security reasons, the total input length is also included in the padding string. Subsequently the resulting string is divided into $t$ blocks $x_{1}$ through $x_{t}$. The hash result is then computed as follows:

$$
\begin{aligned}
H_{0} & =I V \\
H_{i} & =f\left(x_{i}, H_{i-1}\right) \quad i=1,2, \ldots t \\
h(X) & =H_{t} .
\end{aligned}
$$

Here $I V$ is the Initial Value that is fixed for a given hash function and $H_{i}$ is called the chaining variable. In 1999, Merkle and Damgård have proven independently that for this construction the collision resistance of $f$ is a sufficient condition for the collision resistance of $h$. Fixing the $I V$ and appending the total length is therefore known as Merkle-Damgård or MD strengthening $[65,25]$ and the above construction is often referred to as the MD construction.

In 1992, Lai and Massey claimed that finding 2nd preimages for $h$ takes time $2^{n}$ if and only if finding 2 nd preimages for $f$ takes time $2^{n}$ [54], but this result was shown to be incorrect. An important weakness of any hash function is that if one hashes $2^{r}$ messages, finding a (2nd) preimage for one of these takes 
time only $2^{n-r}$. This was already pointed out by Merkle [64]; his solution is to randomize the hash function, i.e., to introduce an additional input (a random parameter).

In the last years, a number of weaknesses have been identified in the MD construction. A first result by Dean et al. [26] was independently discovered and extended by Kelsey and Schneier [51]: if one hashes $2^{b}$ blocks with an iterated hash function, the effort to find a 2 nd preimage is only $r 2^{n / 2+1}+2^{n-b+1}$. It was believed that appending the length in the MD construction would preclude this security degradation with the number $2^{b}$ of blocks (in contrast to a degradation with the number $2^{r}$ of messages). This result disproves the 1992 claim of Lai and Massey mentioned above. A second result by Kelsey and Kohno [50] (known as the herding attack) shows that cheating on commitments is easier than believed: one can commit with a precomputation effort of $2 \cdot 2^{t / 2} \cdot 2^{n / 2}$ to a string $h(x)$; later on, one can choose an arbitrary string $y$ and find in time $2^{n-t}$ strings $z$ and $x^{\prime}$ such that $h\left(y\|z\| x^{\prime}\right)=h(x)$. A third result by Joux shows that if finding a collision for an iterated hash function takes time $T$ (for an ideally secure hash function $T=2^{n / 2}$ ) one can find $2^{s}$ strings hashing to a single value in time $s \cdot T$. As an example, finding a million messages that all hash to the same result requires only twenty times the effort to find a single collision. This result has the surprising corollary that the concatenation of two iterated hash functions $\left(g(x)=h_{1}(x) \| h_{2}(x)\right)$ is only as strong as the strongest of the two hash functions (even if both are independent). If $h_{i}$ is a hash function with an $n_{i}$-bit result $\left(i=1,2\right.$ and w.l.o.g. $\left.n_{1} \geq n_{2}\right)$, finding a collision for $g$ requires time at most $n_{1} \cdot 2^{n_{2} / 2}+2^{n_{1} / 2} \ll 2^{\left(n_{1}+n_{2}\right) / 2}$ and finding a preimage or 2 nd preimage for $g$ requires time at most $n_{1} \cdot 2^{n_{2} / 2}+2^{n_{1}}+2^{n_{2}} \ll 2^{n_{1}+n_{2}}$. If either of the functions is weak, the attacks may work better. This result is particularly relevant since weaknesses have been discovered in several widely used hash functions (cf. infra) and the concatenation construction has been proposed as a robust solution. The main conclusion of this result is that once the collision resistance of our current iterated hash functions breaks down, the other properties are also undermined.

The most widely used hash functions are without any doubt MD5 and SHA-1. MD5 is a 128-bit hash function designed by Rivest in 1991 [75]; it is a strengthened version of MD4. MD5 was one of the first cryptographic algorithms that was designed to be fast on 32-bit processors in software; this would make if very suitable for high-end smart cards today, but on 8-bit machines both are 3-4 times slower than DES. Early cryptanalytic results by den Boer and Bosselaers [27] and Dobbertin [28] indicated that finding collisions for MD5 would require less than $2^{64}$ operations; in spite of the fact that cryptographers advised against using MD5, the algorithm has been widely deployed. The first collisions for MD5 were announced in 2004 by Wang et al. [85], who were able to push the limits on differential attacks by introducing some innovative cryptanalytic techniques; their attack required time $2^{39}$, which corresponds 
to a few hours on a PC. Since then the attack has been further optimized; the best collision search algorithm known in the Summer of 2006 requires on average a few seconds [53]. While this represents a major breakthrough, it is important to note that with about US $\$ 100000$ of hardware, a brute-force collision search for MD5 (or any 128-bit hash function of comparable cost) should take less than one month with the design of van Oorschot and Wiener [84].

In 1995, the US NIST (National Institute for Standards and Technology) has published SHA-1 [34]; it is a strengthened version of SHA, which was published 2 years earlier [33] (SHA is now called SHA-0 by some researchers). While SHA-1 is slower but more secure than MD5, it became very popular for applications that require long term security. In 2005, Wang et al. [86] announced a collision search algorithm for SHA-1 that requires only $2^{63}$ steps. One can expect that collisions for SHA-1 will be found in 2007. In 2005 Joux et al. [48] found collisions for SHA(-0) with complexity $2^{51}$; Wang et al. [87] have developed a more efficient algorithm with complexity $2^{39}$. The implications of the attack on $\mathrm{SHA}(-0)$ are limited, since this algorithm is not deployed.

The collision attack on MD5 is very easy to carry out and the collision attack on SHA-1 is close to practical. Even if both algorithms are widely used, these attacks have not created a cryptographic 'panic reaction.' This is the more surprising since we know from experience that upgrading a cryptographic algorithm can take 3-5 years. A first reason for this moderate response is that the collisions are mainly important for digital signatures that need to provide non-repudiation of origin, that is, to bind a signer to a document. In spite of the development of legislation (e.g., in the framework of the European Directive [32]), very few applications have so far been deployed that require this property. The large majority of applications requires only 2 nd preimage resistance, which is much harder to subvert; breaking 2nd preimage resistance is also required to attack existing digital signatures (that is, signatures that have been placed before the collision attack was published). For MD4, a realistic 2nd preimage attack is known [91] but it only works for a tiny fraction of the message space ( 1 message in $2^{56}$ ), hence it presents no real threat.

The collisions found for the recent attack have a very particular difference in two 512-bit blocks $x_{1} \| x_{2}$ and $x_{1}^{\prime} \| x_{2}^{\prime}$. Only part of the bits in the blocks $x_{i}$ can be chosen by the opponent; the rest of them follow from the attack. However, due to the iterated structure of MD5 and SHA-1, an attacker can choose the beginning and end of the message, hence the colliding messages can be of the form $y\left\|x_{1}\right\| x_{2}\|z, y\| x_{1}^{\prime}\left\|x_{2}^{\prime}\right\| z$ where both $y$ and $z$ can be chosen freely. This is sufficient to produce two colliding executables, two colliding RSA public keys, which have colliding X.509 certificates [57] (no realistic attack scenario is known to exploit this), and two colliding postscript documents [59] or pictures. In the last two cases, one exploits document format with an "if then else" interpreter. It should be clear that it would be unwise to sign any 
document in a format with such a construct, since the document displayed may be completely different depending on the machine or context where it is viewed.

One can expect that the attacks on MD5 and particularly on SHA-1 will be further improved. Therefore it is strongly recommended to replace MD5 immediately for applications involving non-repudiation of origin and as soon as convenient for all other applications. SHA-1 should be removed by 2007 from applications that require non-repudiation of origin (note that NIST recommends to remove SHA-1 as soon as convenient, but replacement is only mandated by 2010). A brute-force collision search for SHA-1 should take in 2010 about 1 year with a budget of US\$50M.

An important question is by which algorithms one should substitute MD5 and SHA-1. A natural replacement for SHA-1 is RIPEMD-160 [20]; this design by Dobbertin et al. seems to resist the current collision attacks and should be sufficient for the next 5-8 years. NIST has also a series of standards ready that offer longer hash results: SHA-256, SHA-224, SHA-384 and SHA-512 [35]; however, the cryptanalytic results on SHA-1 cast some doubt whether shortcut collision attacks may exist on these functions. Another alternative is Whirlpool, a design by Rijmen and Barreto [45]. All these hash functions have been standardized by ISO in IS 10118-3 [45]. A short term solution is to keep using SHA-1 but to randomize the hash function (e.g., Halevi and Krawvzyk [40]). One could also use hash functions based on block ciphers that were already discussed in [19] and that can be found in IS 10118-2 [45].

NIST is considering to organize an open competition for a new hash function standard between 2008 and 2011; several workshops are currently being held to discuss the status of hash function research. One of the research approaches that is being considered is to increase the internal memory to strengthen the protection against structural attacks (e.g., Lucks [59]).

\subsection{Message Authentication Codes (MACs)}

Message Authentication Codes are used to authenticate messages between parties that share a secret key. MACs are widely use in smart card applications, because they are more efficient in terms of performance and memory than digital signature schemes. The most widely used constructions are derived from block ciphers or hash functions.

The most popular MAC algorithm for financial transactions is still CBCMAC. Initially, variants based on DES were used; these have been migrated to triple-DES variants. AES is gradually replacing DES for this application (cf. Sect. 2.3). 
The CBC-MAC construction based on an $n$-bit block cipher can be described as follows. First the input string is padded to a multiple of the block length, and the resulting string is divided into $t n$-bit blocks $x_{1}$ through $x_{t}$.

$$
\begin{aligned}
c_{1} & :=E_{k}\left(x_{0}\right) \\
c_{i} & :=E_{k}\left(x_{i} \oplus c_{i-1}\right), \quad 1<i \leq t .
\end{aligned}
$$

Here $\oplus$ denotes the bitwise exclusive-or operation. Note that - unlike in CBC encryption - no $I V$ value should be used. The recommended variant for use with DES is the ANSI retail MAC [1]: it computes the MAC value with two independent keys $k$ and $k^{\prime}: \operatorname{MAC}_{k}\left(x_{0} \ldots x_{t}\right)=E_{k}\left(E_{k^{\prime}}\left(c_{t}\right)\right)$. For AES, EMAC is the recommended construction: $\operatorname{MAC}_{k}\left(x_{0} \ldots x_{t}\right)=E_{k^{\prime}}\left(c_{t}\right)$. Here $k^{\prime}$ is a key derived from $k$.

NIST has published yet another variant under the name of CMAC [68] (CMAC was previously called OMAC [47], which is in turn an optimization of XCBC [14]). CMAC modifies the last computation in CBC-MAC by exoring $k 2$ or $k 3$ to $x_{t}$. The key $k 2$ is chosen when the last block $x_{t}$ requires no padding (i.e., it is of length $n$ ), while $k 3$ is chosen otherwise. The keys $k 2$ and $k 3$ are computed as $k 2=$ ' 2 ' $\cdot E_{k}\left(0^{n}\right)$ and $k 3={ }^{\prime} 4$ ' $\cdot E_{k}\left(0^{n}\right)$ where $0^{n}$ denotes the $n$-bit all zero string, ' 2 ' and ' 4 ' are two elements of the finite field $\operatorname{GF}\left(2^{n}\right)$, and "." represents multiplication in the finite field $\operatorname{GF}\left(2^{n}\right)$. A potential weakness related to the use of CMAC is that the value $E_{k}\left(0^{n}\right)$ is employed in some financial protocols for key confirmation.

On the Internet, HMAC is by far the most popular construction [5]; its security analysis has been refined recently by Bellare [4]. Unfortunately, the recent cryptanalytic results on MD4 and MD5 have some implications on its practical security: HMAC-MD4 has been broken by Contini and Yin [21], and some doubts have been cast on HMAC-MD5 [21,52]. For the time being HMACSHA-1 is still fine, but it seems that the MDx-MAC construction of [74] is more resistant to the weaknesses discovered in MD5 and SHA-1.

In the past five years there has been a growing interest in unconditionally secure MAC algorithms. They were introduced as authentication codes by Simmons [82] and more practical constructions were known as universal hash functions (following Carter and Wegman [89]). If they are combined with a block cipher (such as AES) or a pseudo-random function (such as HMAC), the unconditional security is lost, but they result in MAC algorithms that are very efficient and elegant. UMAC [13] is about 10 times faster than CBCMAC based on AES or HMAC-SHA-1, but it offers a limited key agility and has a rather large Random Access Memory (RAM) requirement. Bernstein's Poly1305-AES [10] is one of the constructions based on polynomial universal hashing. It is only three times faster than AES, but it has a better key agility than UMAC and requires less RAM. 


\subsection{Block Ciphers}

Block ciphers are a widely used cryptographic primitive; they are a flexible building block for many cryptographic applications. This includes the original goal of encryption (in CBC, CFB, OFB or CTR mode), but they can also be used to construct MAC algorithms (cf. Section 2.2), hash functions, pseudorandom functions and one-way functions.

The US government has published in the 1970s the DES algorithm, which has a 64-bit block length and a 56-bit key. The approval by the US government and its free use were deciding factors in its success. However, in the 1990s it became obvious that the 56-bit key size of DES was no longer adequate (a US\$ 1 million machine today would recover a DES key in less than a minute - the same design would have taken 3 hours in 1993 [90]). The financial world started moving towards triple-DES in the late 1990s, and in 2004 NIST announced that DES was no longer adequate and published a triple-DES specification [67]; two-key triple-DES is approved until 2009, while three-key triple-DES is deemed to be adequate until 2030. The modes for triple-DES have been defined in ANSI $\mathrm{X} 9.52[2]$.

In 1997, NIST started an open competition to find a replacement for the DES. The AES algorithm has a block of length of 128 bits, and should support keys lengths of 128, 192 and 256 bits. In October 2000 NIST selected the Rijndael algorithm (designed by the Belgian cryptographers Vincent Rijmen and Joan Daemen) as the AES algorithm [24,36]. In 2003, the US government announced that it would also allow the use of AES for secret data, and even for top secret data; the latter applications require key lengths of 192 or 256 bits. AES is a rather elegant and mathematical design, that among the five finalists offered the best combination of security, performance, efficiency, implementability and flexibility. AES allows for efficient implementations on 32-bit architectures (15 cycles/byte on a Pentium III), but also for compact implementations on 8bit smart cards. Moreover, hardware implementations of AES offer good tradeoffs between size and speed. AES has been taken up quickly by many standards and implementations; by the end of 2006 more than 400 AES implementations have been validated by the US government.

Several researchers have claimed that the mathematical structure of AES could be the source of weaknesses against algebraic attacks $[22,66]$. However, to this date none of the proposed attack approaches has been shown to be viable, even to break highly reduced version of AES. The most powerful attacks against AES have not been pure mathematical attacks, but timing attacks based on cache effects - this kind of attack applies in principle to any cryptographic algorithm implementation that uses tables. It is not possible to present a complete overview of all these attacks within the scope of this paper; some 
useful references include the work of Tsunoo et al. [83], Bernstein [10] and Osvik et al. [69].

\subsection{Stream Ciphers}

Additive stream ciphers have been the work horse of symmetric crypto until the 1980s. They take as input a short secret key and a public initialization value $I V$ and stretch this to a long string that can be simply added to the plaintext to yield the ciphertext. This implies that the encryption transformation depends on the location in the plaintext, yet it is very simple. Hardware oriented stream ciphers typically operate on short data units (bits or bytes) and have a small footprint. The initialization value $I V$ serves for resynchronization purposes. Both the $I V$ and the internal memory need to be sufficiently large to resist time-memory-data tradeoffs (see for example $[41,55]$ ).

From the 1960s to the late 1980s, most stream ciphers were based on Linear Feedback Shift Registers (LFSRs) that are optimal for hardware implementations (see for example Rueppel [79] and Menezes et al. [63]). However, it has become clear that most LFSR-based stream ciphers are much less secure than expected; powerful new attacks include fast correlation attacks [62] and algebraic attacks [23]. Notable cryptanalytic successes are the attack by Barkan and Biham [3] on A5/1 (the stream cipher used in GSM) and the attack by $\mathrm{Lu}$ et al. [58] on E0 (the stream cipher used in Bluetooth). Both attacks are realistic attacks on widely used algorithms.

RC4 has been designed in 1987 by Ron Rivest for efficient software encryption on 8-bit machines. RC4 was a trade secret, but leaked out in 1994; currently it is widely used in browsers (SSL/TLS protocol). While several statistical weaknesses have been identified in RC4 $[37,70]$ the algorithm still seems to resist key recovery attacks.

In the last decade, fast stream ciphers have been proposed that are oriented towards 32-bit and 64-bit processors. Two stream ciphers that have been included into the ISO standard are MUGI [88] and SNOW [30]; a strengthened variant of SNOW will be included as second algorithm in 3GSM. The EU Network of Excellence ECRYPT [29] is currently running an open competition eSTREAM with as goal to identify promising stream ciphers that are either very fast in software (128-bit key and 64 or 128-bit $I V$ ) or that offer a low footprint in hardware (80-bit key and 32 or 64-bit $I V$ ). By early 2008 the eSTREAM initiative will complete its shortlist of promising stream ciphers. 


\subsection{Authenticated or Unforgeable Encryption}

Most applications need a secure channel between sender and receiver; such a channel requires both confidentiality and data authentication. As can be seen from the discussion above, separate primitives were introduced for each of these properties. However, it is not so hard to show that confidentiality protection without data authentication can lead to serious problems; in particular, such a scheme is vulnerable to a chosen ciphertext attack in which the opponent uses decryption queries to learn information on the plaintext. The first approach to achieve both properties was to introduce redundancy to the plaintext before encryption in order to achieve both goals, but this is clearly not adequate. A first formalization of unforgeable encryption was published by Katz and Yung [49]. Bellare and Namprempre [6] showed that if the MAC algorithm satisfies a strong security requirement (namely strong unforgeability), the best generic solution is to apply a MAC algorithm to the ciphertext (the so-called Encrypt-then-MAC model), which is the option chosen by IPsec. Other alternatives (MAC-then-Encrypt of SSL/TLS and Encrypt and MAC of SSH) can also be shown to be secure, but they require a specific rather than a generic analysis (e.g., taking into account the specific encryption mode).

The above schemes require both an encryption algorithm and a MAC algorithm. Jutla showed that it was possible to achieve both properties at a much lower cost; for this purpose he introduced in 2000 two modes, the IACBC (Integrity-Aware Cipher Block Chaining) and IAPM (Integrity-Aware Parallelizable Mode). Gligor and Donescu proposed the XCBC and XECB schemes in [39]. Rogaware et al. [77] introduced an optimized version of IAPM called the OCB mode (Offset CodeBook). These schemes require an overhead of less than $10 \%$ over CBC encryption and offer some attractive features; for example, some of them are fully parallellizeable. An important non-technical disadvantage is that all these schemes are encumbered by patents, which has been a barrier to their adoption.

As a consequence of this patent issue, several alternative schemes have been introduced that are slower than these schemes, but that are free. As examples, we mention the EAX [9] and the GCM [61] (Galois Counter Mode). Both use the CTR mode for encryption; EAX uses CBC-MAC, while GCM uses a MAC algorithm based on polynomial universal hashing. For a more detailed overview of authenticated encryption schemes, see the overview article by Black [12]. 


\section{$3 \quad$ Public Key Algorithms}

\section{$3.1 R S A$}

RSA, invented by Rivest, Shamir and Adleman in 1978 [76] is still the most widely used public key algorithm (the RSA patent has expired in 2000). The RSA encryption operation is written as $C=P^{e} \bmod N$ and the decryption is computed as $P=C^{d} \bmod N$. Here the encryption and decryption exponent are related by $e \cdot d=1 \bmod \operatorname{lcm}(p-1, q-1)$, with $N=p \cdot q$. The security of RSA is based on the fact that it is relatively easy to find two large prime numbers $p$ and $q$, but no efficient methods are known to factor their product $N$. Note that the security of RSA is based on the fact that extracting random modular eth roots $\bmod N$ is hard. This problem could be easier than factoring $N$ (it cannot be harder); surprisingly, whether or not it is easier is still an open problem.

The best known algorithm to factor an RSA modulus $N$ is the General Number Field Sieve; it has (heuristic) asymptotic complexity

$$
O\left(\exp \left[(1.923+o(1)) \cdot(\ln N)^{1 / 3} \cdot(\ln \ln N)^{2 / 3}\right]\right) .
$$

Lenstra and Verheul have related this complexity to breaking symmetric keys and computing discrete logarithms in [56] (see also the ECRYPT report on this topic [29]). The current factoring record (achieved in May 2005) is 200 digits or 663 bits. The recommended minimum size for an RSA modulus in 2006 is 1024 bits; factoring such a modulus requires approximately $2^{80}$ steps. Shamir and Tromer [80] propose a hardware design that would need an R\&D effort of US $\$ 20 \mathrm{M}$. The hardware cost to factor a 512-bit modulus in ten minutes would be US\$ 10000; a 768-bit modulus could be factored with a similar budget in 95 days; factoring a 1024-bit modulus in 1 year would require a hardware investment of US $\$ 10 \mathrm{M}$. Note that these cost estimates do not include the linear algebra step. These estimates show that for long-term security (10-15 years), an RSA modulus of 1536 to 2048 bits is recommended.

Textbook RSA has other weaknesses (see [18] for details). For example, RSA for small arguments is not secure: $-1,0$ and 1 are always fixed points and if $P^{e}<N$ extracting a modular eth root simplifies to extracting a natural eth root, which is an easy problem. In addition, RSA is multiplicative, which means that the product $\bmod N$ of two ciphertexts will decrypt to the product of the corresponding plaintexts.

The standard PKCS\#1v1.5 specifies a padding method for encryption and signing with the RSA algorithm. For encryption, the format consists of the 
following sequence: a byte equal to 00 , a byte equal to 02 , at least 8 non-zero padding bytes, a byte 00 , and the plaintext. Note that the RSA assumption states that extracting random modular eth roots is hard, which means that one should map the plaintext space in a uniform way to the interval $[0, n[$; it is clear that PKCS\#1v1.5 is quite far from this goal. This has been exploited by Bleichenbacher [15] to recover the plaintext corresponding to a selected ciphertext using a chosen ciphertext attack (in which encryptions of different but related ciphertexts are obtained); more specifically, Bleichenbacher's attack only needs to know whether the plaintext is of the right format (it is based on the error messages). In 1993, Bellare and Rogaway published the OAEP (Optimal Asymmetric Encryption) transform, together with a security proof [7]. This proof essentially states that if someone can decrypt a challenge ciphertext without knowing the secret key, he can extract random modular eth roots. The proof is in the random oracle model, which means that the hash functions used in the OAEP construction are assumed to be perfectly random. However, seven years later Shoup pointed out that the proof was wrong [81]; the error has been corrected for by Fujisaki et al. in [38], but the resulting reduction is not very meaningful, that is, the coupling between the two problems is not very tight in this new proof. Moreover, Manger showed that a careful implementation is necessary, since otherwise a chosen ciphertext attack based on error messages may still apply [60]. Currently the cryptographic community believes that the best way of using RSA is the RSA-KEM mode [73]: this is a so-called hybrid mode in which RSA is only used to transfer a session key, while the plaintext is encrypted using a symmetric algorithm with this key.

For RSA PKCS\#1v1.5 signatures, no practical attack is known, even if this padding format is again very far from random. The RSA signing operation is applied to the following sequence: a byte equal to 00, a byte equal to 01 , a series of bytes equal to FF, a byte 00, and the hash value (with some ASN.1 prepended). At the rump session of Crypto 2006, Bleichenbacher showed that many implementations of RSA signature verifications stop at the end of the hash value. This opens the possibility to append a large random string $S$ (and shorten the series of FF bytes accordingly). It is very easy to choose $S$ such that the complete string is a perfect cube, and extracting cube roots over the integers is easy. This means that one can forge any signature for $e=3$ without knowing the private key; even better, this forged signature works for any modulus $N$ that is large enough. A variant of the attack is based on the fact that some verification software ignores the content of the ASN.1 string. These attacks can be precluded by implementing a correct verification, which consists of checking that the hash value is right aligned or alternatively by regenerating the whole block as the signer does and checking that it is correct. The problem is however that as a signer may not be able to influence the verification software, hence it is better to increase the verification exponent to $2^{16}+1$. Implementations that were reported to be vulnerable to this problem include OpenSSL, Mozilla NSS, and GnuTLS. A better solution is to use RSA- 
PSS [8], which has been included together with OAEP in PKCS\#1 v2.1. Even if the scheme dates back to 1996 and the standard to 2002, so far implementors seem to be reluctant to upgrade to the more robust algorithms.

For performance reasons, the RSA private key operations (decryption and signing) are often executed using the Chinese remainder theorem. This means that they are computed $\bmod p$ and $\bmod q$ and that both results are combined to recover the result $\bmod N$. One of the most important vulnerabilities of RSA in practice is the observation by Boneh et al. [17]: if a transient fault is introduced in the calculation $\bmod p$ or $\bmod p($ but not both), one can recover $p$ and $q$. Making an implementation robust against these powerful fault attacks is non-trivial.

An important lesson that can be drawn from this is that it is surprisingly difficult to use RSA correctly: it has taken the cryptographic community more than 20 years to learn how to do this. The most efficient solutions still rely on the random oracle model, and it is an important problem how one can use RSA efficiently without this assumption.

\subsection{Elliptic Curve Cryptography (ECC)}

Elliptic curve cryptography (ECC) is a public-key primitive that is increasingly important as alternative to RSA. The standards (e.g., [46,42]) support both elliptic curves over $F_{p}$ with $p$ prime and $F_{2^{m}}$ with $m$ prime. The first curves can take advantage from an arithmetic coprocessor for RSA if available, while the latter allow for very compact hardware implementations.

An important advantage of elliptic curves are the shorter key lengths. Based on the best known algorithms today, one can estimate that 166-bit elliptic curves correspond to 1024-bit RSA, and 206-bit elliptic curves correspond to 2048-bit RSA (see the ECRYPT report [29]). For these bit-lengths, signing is six (resp. 19) times faster with elliptic curves, but verifying a signature is seven (resp. five) times faster with RSA. Moreover, very compact hardware implementations of ECC have been developed.

Even if ECC was proposed in 1985, the market was initially reluctant to move towards this new and more complex primitive. However, recently ECC has been adopted by the governments of Austria, Germany and the USA and are gaining more widespread acceptance. The main attraction lies clearly in the shorter key lengths; this advantage over RSA will increase over time. 


\section{Conclusions}

In the past five years the world of cryptographic algorithms has changed substantially. As smart cards become more powerful, the distinction between cryptographic algorithms for smart cards and cryptographic algorithms for high end machines is disappearing.

The AES has quickly become a worldwide standard in many applications. Some powerful new attack techniques have made new victims in the area of hash functions and stream ciphers. In addition, side channel attacks have become an important area of research: they currently strongly influence hardware and software implementations, but at the cost of a decreased performance. One can expect that in the future some algorithms will be re-designed from scratch so that implementing these algorithms in a secure way is easier.

In addition to new attacks, new security proofs and models have been developed, that increase our understanding in areas such as modes for confidentiality and authenticated encryption and padding methods for RSA and ECC.

In both cases (new attacks and new models and designs), there is a need for efficient and secure procedures to upgrade and retire cryptographic algorithms. However, even if we live in a world in which the environment can change in days or months, replacing a cryptographic algorithm still takes many years. System designers need to build systems that are agnostic to the cryptographic algorithm and that allow for fast and secure key length and algorithm upgrades.

\section{References}

[1] ANSI X9.19, Financial Institution Retail Message Authentication, American Bankers Association, August 13, 1986.

[2] ANSI X9.52, Triple Data Encryption Algorithm Modes of Operation, American Bankers Association, 1998.

[3] E. Barkan, E. Biham, N. Keller, Instant ciphertext-only cryptanalysis of GSM encrypted communication, Advances in Cryptology, Proceedings Crypto'03, LNCS 2729, D. Boneh, Ed. (Springer-Verlag, 2003) 600-616.

[4] M. Bellare, New proofs for NMAC and HMAC: Security without collisionresistance, Advances in Cryptology, Proceedings Crypto'06, LNCS 4117 C. Dwork, Ed. (Springer Verlag, 2006) 602-619. 
[5] M. Bellare, R. Canetti, H. Krawczyk, Keying hash functions for message authentication, Advances in Cryptology, Proceedings Crypto'96, LNCS 1109 N. Koblitz, Ed. (Springer-Verlag, 1996) 1-15.

[6] M. Bellare, C. Namprempre, Authenticated encryption: Relations among notions and analysis of the generic composition paradigm, Advances in Cryptology, Proceedings Asiacrypt'00, LNCS 1976, T. Okamoto, Ed. (SpringerVerlag, 2000) 531-545.

[7] M. Bellare, P. Rogaway, Random oracles are practical: A paradigm for designing efficient protocols, Proceedings ACM Conference on Computer and Communications Security (ACM Press 1993) 62-73.

[8] M. Bellare, P. Rogaway, The exact security of digital signatures - How to sign with RSA and Rabin, Advances in Cryptology, Proceedings Eurocrypt'96, LNCS 1070, U. Maurer, Ed. (Springer-Verlag, 1996) 399-416.

[9] M. Bellare, P. Rogaway, D. Wagner, The EAX mode of operation, Fast Software Encryption, LNCS 3017, B.K. Roy, W. Meier, Eds. (Springer-Verlag, 2004) 389407.

[10] D.J. Bernstein, The Poly1305-AES message-authentication code, Fast Software Encryption, LNCS 355\%, H. Gilbert, H. Handschuh, Eds. (Springer-Verlag, 2005) 32-49.

[11] D.J. Bernstein, Cache-timing attacks on AES, preprint, 2005, http://cr.yp. to/papers.html\#cachetiming

[12] J. Black, Authenticated encryption, Encyclopedia of Cryptography and Security H. van Tilborg, Ed. (Springer-Verlag, 2005) 11-21.

[13] J. Black, S. Halevi, H. Krawczyk, T. Krovetz, P. Rogaway, UMAC: Fast and secure message authentication, Advances in Cryptology, Proceedings Crypto'99, LNCS 1666, M. Wiener, Ed. (Springer-Verlag 1999) 216-233.

[14] J. Black, P. Rogaway, CBC-MACs for arbitrary length messages, Advances in Cryptology, Proceedings Crypto'00, LNCS 1880, M. Bellare, Ed. (SpringerVerlag, 2000) 197-215.

[15] D. Bleichenbacher, Chosen ciphertext attacks against protocols based on the RSA encryption standard PKCS \#1, Advances in Cryptology, Proceedings Crypto'98, LNCS 1462, H. Krawczyk, Ed. (Springer-Verlag 1998) 1-12.

[16] D. Bleichenbacher, Forging some RSA signatures with pencil and paper, Presented at the Rump Session of Crypto 2006.

[17] D. Boneh, R. DeMillo, R. Lipton, On the importance of checking cryptographic protocols for faults, Advances in Cryptology, Proceedings Eurocrypt'97, LNCS 1233 W. Fumy, Ed. (Springer-Verlag, 1997) 37-51.

[18] D. Boneh, A. Joux, P.Q. Nguyen, Why textbook ElGamal and RSA encryption are insecure, Advances in Cryptology, Proceedings Asiacrypt'00, LNCS 1976, T. Okamoto, Ed. (Springer-Verlag, 2000) 30-43. 
[19] J. Borst, B. Preneel, V. Rijmen, Cryptography on smart cards, Computer Networks Vol. 36, No. 4 (2001) 423-435.

[20] A. Bosselaers, H. Dobbertin, B. Preneel, The RIPEMD-160 cryptographic hash function, Dr. Dobb's Journal, Vol. 22, No. 1 (January 1997) 24-28.

[21] S. Contini, Y.L. Lin, Forgery and partial key recovery attacks on HMAC and NMAC using hash collisions Advances in Cryptology, Proceedings Asiacrypt'06, LNCS 4284, X. Lai, Ed. (Springer-Verlag, 2006) 37-53.

[22] N. Courtois, J. Pieprzyk, Cryptanalysis of block ciphers with overdefined systems of equations. Advances in Cryptology, Proceedings Asiacrypt'02, LNCS 2501, Y. Zheng, Ed. (Springer-Verlag, 2002) 267-287.

[23] N. Courtois, W. Meier, Algebraic attacks on stream ciphers with linear feedback, Advances in Cryptology, Proceedings Eurocrypt'03, LNCS 2656, E. Biham, Ed. (Springer-Verlag, 2003) 345-359.

[24] J. Daemen, V. Rijmen, The Design of Rijndael. AES - The Advanced Encryption Standard, Springer-Verlag (2001).

[25] I.B. Damgård, A design principle for hash functions, Advances in Cryptology, Proceedings Crypto'89, LNCS 435, G. Brassard, Ed. (Springer-Verlag, 1990) 416-427.

[26] R.D. Dean, Formal aspects of mobile code security, PhD thesis, Princeton University, January 1999.

[27] B. den Boer, A. Bosselaers, Collisions for the compression function of MD5, Advances in Cryptology, Proceedings Eurocrypt'93, LNCS 765, T. Helleseth, Ed. (Springer-Verlag, 1994) 293-304.

[28] H. Dobbertin, The status of MD5 after a recent attack, CryptoBytes, Vol. 2, No. 2, Summer 1996, 1-6.

[29] EU Network of Excellence ECRYPT, http://www.ecrypt.eu.org

[30] P. Ekdahl, T. Johansson, A new version of the stream cipher SNOW, Selected Areas in Cryptography, SAC'02, LNCS 2595, K. Nyberg, H.M. Heys, Eds. (Springer-Verlag, 2003) 47-61.

[31] Electronic Frontier Foundation, Cracking DES, Secrets of Encryption Research, Wiretap Politics 83 Chip Design, (O'Reilly \& Associates, Sebastopol, 1998). Source code of the implementation described in the book can be downloaded from https://www.cosic.esat.kuleuven.ac.be/des/.

[32] EU Directive 1999/93/EC, Community framework for electronic signatures, 13 December 1999.

[33] FIPS 180, Secure Hash Standard, Federal Information Processing Standard (FIPS), Publication 180, NIST, U.S. Dept. of Commerce (May 11, 1993).

[34] FIPS 180-1, Secure Hash Standard, Federal Information Processing Standard (FIPS), Publication 180-1, NIST, U.S. Dept. of Commerce (April 17, 1995). 
[35] FIPS 180-2, Secure Hash Standard, Federal Information Processing Standard (FIPS), Publication 180-2, NIST U.S. Dept. of Commerce (August 26, 2002) (Change notice 1 published on December 1, 2003).

[36] FIPS 197, Advanced Encryption Standard, Federal Information Processing Standard, NIST, U.S. Dept. of Commerce (November 26, 2001).

[37] S. Fluhrer, I. Mantin, A. Shamir, Weaknesses in the key scheduling algorithm of RC4, Selected Areas in Cryptography, SAC'01, LNCS 2259, S. Vaudenay, A. Youssef, Eds. (Springer-Verlag 2001) 1-24.

[38] E. Fujisaki, T. Okamoto, D. Pointcheval, J. Stern, RSA-OAEP is secure under the RSA assumption, Advances in Cryptology, Proceedings Crypto'01, LNCS 2139, J. Killian, Ed. (Springer-Verlag, 2001) 260-274.

[39] V.D. Gligor, P. Donescu, Fast encryption and authentication: XCBC encryption and XECB authentication modes, Fast Software Encryption, LNCS 2355, M. Matsui, Ed. (Springer-Verlag, 2002) 92-108.

[40] S. Halevi, H. Krawczyk, Strengthening digital signatures via randomized hashing, Advances in Cryptology, Proceedings Crypto'06, LNCS 4117 C. Dwork, Ed. (Springer Verlag, 2006) 41-59.

[41] J. Hong, P. Sarkar, New applications of time memory data tradeoffs, Advances in Cryptology, Proceedings Asiacrypt'05, LNCS 3788, B.K. Roy, Ed. (SpringerVerlag, 2005) 353-372.

[42] IEEE P1363, Standard Specifications for Public Key Cryptography, 2000.

[43] ISO/IEC 7816, Information technology - Identification cards - Integrated circuit(s) cards with contacts - Part 4: Interindustry commands for interchange, 1997.

[44] ISO/IEC 9797, Information technology - Security techniques - Message Authentication Codes (MACs), Part 1: Mechanisms using a block cipher, 1999, Part 2: Mechanisms using a hash-function, 2000.

[45] ISO/IEC 10118, Information technology - Security techniques - Hash-functions, Part 1: General, 2000, Part 2: Hash-functions using an $n$-bit block cipher algorithm, 2000, Part 3: Dedicated hash-functions, 2003. Part 4: Hash-functions using modular arithmetic, 1998.

[46] ISO/IEC 14888-3, Information technology - Security techniques - Digital signatures with appendix, Part 3: Certificate-based mechanisms, 1998.

[47] T. Iwata, K. Kurosawa, OMAC: One key CBC MAC, Fast Software Encryption, LNCS 2887, T. Johansson, Ed. (Springer-Verlag, 2003) 129-153.

[48] A. Joux, Multicollisions in iterated hash functions. Application to cascaded constructions. Advances in Cryptology, Proceedings Crypto'04, LNCS 3152 M.K. Franklin, Ed., (Springer-Verlag, 2004) 306-316. 
[49] J. Katz, M. Yung, Unforgeable encryption and chosen ciphertext secure modes of operation, Fast Software Encryption, LNCS 1978, B. Schneier, Ed. (SpringerVerlag, 2001) 284-299.

[50] J. Kelsey, T. Kohno, Herding hash functions and the Nostradamus attack, Advances in Cryptology, Proceedings Eurocrypt'06, LNCS 4004, S. Vaudenay, Ed. (Springer-Verlag, 2006) 183-200.

[51] J. Kelsey, B. Schneier, Second preimages on $n$-bit hash functions for much less than $2^{n}$ work, Advances in Cryptology, Proceedings Eurocrypt'05, LNCS 3494, R. Cramer, Ed., (Springer-Verlag, 2005) 474-490.

[52] J. Kim, A. Biryukov, B. Preneel, S. Hong, On the Security of HMAC and NMAC Based on HAVAL, MD4, MD5, SHA-0 and SHA-1 Security in Communication Networks, LNCS 4116, R. De Prisco, M. Yung, Eds. (Springer-Verlag, 2006) $242-256$.

[53] V. Klima, Tunnels in hash functions: MD5 collisions within a minute, IACR Eprint Server, 2006/105, http://eprint.iacr.org.

[54] X. Lai, J.L. Massey, Hash functions based on block ciphers, Advances in Cryptology, Proceedings Eurocrypt'92, LNCS 658, R.A. Rueppel, Ed. (SpringerVerlag, 1993) 55-70.

[55] J. Lano, Cryptanalysis and Design of Synchronous Stream Ciphers, PhD Thesis, COSIC, K.U.Leuven, June 2006.

[56] A.K. Lenstra, E.R. Verheul, Selecting cryptographic key sizes, Journal of Cryptology, Vol. 14, No. 4 (2001) 255-293.

[57] A.K. Lenstra, B. de Weger, On the possibility of constructing meaningful hash collisions for public keys. Information Security and Privacy, Proceedings ACISP'05, LNCS 3574, C. Boyd, J.M. González Nieto Eds. (Springer-Verlag, 2005) 267-279

[58] Yi Lu, W. Meier, S. Vaudenay, The conditional correlation attack: A practical attack on Bluetooth encryption, Advances in Cryptology, Proceedings Crypto'05, LNCS 3621, V. Shoup, Ed. (Springer-Verlag, 2005) 97-117.

[59] S. Lucks, A failure-friendly design principle for hash functions, Advances in Cryptology, Proceedings Asiacrypt'05, LNCS 3788, B.K. Roy, Ed. (SpringerVerlag, 2005) 474-494.

[60] J. Manger, A chosen ciphertext attack on RSA optimal asymmetric encryption padding (OAEP) as standardized in PKCS\#1 v2.0, Advances in Cryptology, Proceedings Crypto'01, LNCS 2139, J. Killian, Ed. (Springer-Verlag, 2001) 230238.

[61] D. McGrew, J. Viega, The security and performance of the Galois/Counter Mode (GCM) of pperation, Progress in Cryptology, Indocrypt04, LNCS 3348 A Canteaut, K. Viswanathan, Eds. (Springer-Verlag, 2004), 343-355. 
Full paper http://eprint.iacr.org/2004/193/ Submission to NIST. http: //csrc.nist.gov/CryptoToolkit/modes/proposedmodes/gcm/gcm-spec. pdf, January 2004.

[62] W. Meier, O. Staffelbach, Fast correlation attacks on stream ciphers, Journal of Cryptology, Vol. 1, No. 3 (1989) 159-176.

[63] A.J. Menezes, P.C. van Oorschot, S.A. Vanstone, Handbook of Applied Cryptography (CRC Press, 1997).

[64] R. Merkle, Secrecy, Authentication, and Public Key Systems, UMI Research Press, 1979.

[65] R. Merkle, One way hash functions and DES, Advances in Cryptology, Proceedings Crypto'89, LNCS 435, G. Brassard, Ed. (Springer-Verlag, 1990) 428-446.

[66] S. Murphy, M.J.B. Robshaw, Essential algebraic structure within the AES, Advances in Cryptology, Proceedings Crypto'02, LNCS 2442, M. Yung, Ed. (Springer-Verlag, 2002) 1-16.

[67] NIST Special Publication 800-67, Recommendation for the Triple Data Encryption Algorithm (TDEA) Block Cipher, May 2004

[68] NIST Special Publication 800-38B, Recommendation for Block Cipher Modes of Operation: The CMAC Mode for Authentication, May 2005.

[69] D. Osvik, A. Shamir, E. Tromer, Cache attacks and countermeasures: The case of AES, Topics in Cryptology - The Cryptographers' Track at the RSA Conference 2006, LNCS 3860, D. Pointcheval, Ed. (Springer-Verlag 2006) 1-20. Extended version at www.wisdom.weizmann.ac.il/ tromer/papers/cache. pdf

[70] S. Paul, B. Preneel, Analysis of non-fortuitous predictive states of the RC4 key stream Generator, Progress in Cryptology, Indocrypt'04, LNCS 2904, T. Johansson, S. Maitra, Eds. (Springer-Verlag 2003) 30-47.

[71] E. Petrank, C. Rackoff, CBC MAC for real-time data sources, Journal of Cryptology, Vol. 13, No. 3 (2000) 315-338.

[72] B. Preneel, Cryptographic primitives for information authentication - state of the art, State of the Art in Applied Cryptography, LNCS 1528, B. Preneel, V. Rijmen, Eds., (Springer-Verlag, 1998) 50-105.

[73] B. Preneel, A. Biryukov, C. De Cannière, S.B. Örs, E. Oswald, B. Van Rompay, L. Granboulan, E. Dottax, G. Martinet, S. Murphy, A. Dent, R. Shipsey, C. Swart, J. White, M. Dichtl, S. Pyka, M. Schafheutle, P. Serf, E. Biham, E. Barkan, Y. Braziler, O. Dunkelman, V. Furman, D. Kenigsberg, J. Stolin, JJ. Quisquater, M. Ciet, F. Sica, H. Raddum, L. Knudsen, M. Parker, Final report of NESSIE, New European Schemes for Signatures, Integrity, and Encryption, LNCS Springer-Verlag, in print. 
[74] B. Preneel, P.C. van Oorschot, MDx-MAC and building fast MACs from hash functions, Advances in Cryptology, Proceedings Crypto'95, LNCS 963 D. Coppersmith, Ed. (Springer-Verlag, 1995) 1-14.

[75] R.L. Rivest, The MD5 message-digest algorithm, Request for Comments (RFC) 1321, Internet Activities Board, Internet Privacy Task Force, April 1992.

[76] R.L. Rivest, A. Shamir, L. Adleman, A method for obtaining digital signatures and public-key cryptosystems, Communications ACM, Vol. 21, No. 2 (1978) $120-126$.

[77] P. Rogaway, M. Bellare, J. Black, T. Krovetz, OCB: A block-cipher mode of operation for efficient authenticated encryption, ACM Conference on Computer and Communications Security (ACM Press 2001) 195-205.

[78] P. Rogaway, T. Shrimpton, Cryptographic hash function basics: Definitions, implications, and separations for preimage resistance, second-preimage resistance, and collision resistance, Fast Software Encryption 2004, LNCS 3017 B.K. Roy, W. Meier, Eds. (Springer-Verlag, 2004), 371-388.

[79] R.A. Rueppel, Analysis and Design of Stream Ciphers (Springer-Verlag, 1986).

[80] A. Shamir, E. Tromer, Factoring large numbers with the TWIRL device Advances in Cryptology, Proceedings Crypto'03, LNCS 2729, D. Boneh, Ed. (Springer-Verlag, 2003) 1-26.

[81] V. Shoup, OAEP reconsidered, Advances in Cryptology, Proceedings Crypto'01, LNCS 2139, J. Killian, Ed. (Springer-Verlag, 2001) 239-259.

[82] Contemporary Cryptology: The Science of Information Integrity, G.J. Simmons, Ed. (IEEE Press, 1991).

[83] Y. Tsunoo, T. Saito, T. Suzaki, M. Shigeri, H. Miyauchi, Cryptanalysis of DES implemented on computers with cache, Cryptographic Hardware and Embedded Systems 2003, LNCS 2779, C.D. Walter, Ç.K. Koç, C. Paar, Eds. (SpringerVerlag 2003) 62-76.

[84] P.C. van Oorschot, M. Wiener, Parallel collision search with cryptanalytic applications, Journal of Cryptology, Vol. 12, No. 1 (1999) 1-28.

[85] X. Wang, H. Yu, How to break MD5 and other hash functions, Advances in Cryptology, Proceedings Eurocrypt'05, LNCS 3494, R. Cramer, Ed. (SpringerVerlag, 2005) 19-35.

[86] X. Wang, Y.L. Lin, H. Yu, Finding collisions in the ful SHA-1, Advances in Cryptology, Proceedings Crypto'05, LNCS 3621, V. Shoup, Ed. (SpringerVerlag, 2005) 17-36.

[87] X. Wang, H. Yu, Y.L. Lin, Efficient collision search attacks on SHA-0, Advances in Cryptology, Proceedings Crypto'05, LNCS 3621, V. Shoup, Ed. (SpringerVerlag, 2005) 1-16. 
[88] D. Watanabe, S. Furuya, H. Yoshida, K. Takaragi, B. Preneel, A new keystream generator MUGI, Fast Software Encryption, LNCS 2365, J. Daemen, V. Rijmen, Eds. (Springer-Verlag, 2002) 179-194.

[89] M.N. Wegman, J.L. Carter, New hash functions and their use in authentication and set equality, Journal of Computer and System Sciences, Vol. 22, No. 3 (1981) 265-279.

[90] M.J. Wiener, Efficient DES key search, Presented at the Rump Session of Crypto'93. Reprinted in Practical Cryptography for Data Internetworks, W. Stallings, Ed. (IEEE Computer Society, 1996) 31-79.

[91] H. Yu, G. Wang, G. Zhang, X. Wang, The Second-Preimage Attack on MD4, Cryptology and Network Security, CANS 2005, LNCS 3810, Y. Desmedt, H. Wang, Y. Mu, Y. Li, Eds. (Springer-Verlag, 2005) 1-12. 